\title{
The Social Appearance Anxiety Scale in Italian Adolescent Populations: Construct Validation and Group Discrimination in Community and Clinical Eating Disorders Samples
}

ARTICLE in CHILD PSYCHIATRY AND HUMAN DEVELOPMENT · MAY 2015

Impact Factor: 1.93 · DOI: 10.1007/s10578-015-0551-1 · Source: PubMed

READS

91

8 AUTHORS, INCLUDING:

\section{Antonios Dakanalis}

Università degli Studi di Milano-Bicocca 60 PUBLICATIONS 259 CITATIONS

SEE PROFILE

\section{Giuseppe Riva}

Catholic University of the Sacred Heart 601 PUBLICATIONS 6,906 CITATIONS

SEE PROFILE

\section{Giuseppe Carrà}

University College London

124 PUBLICATIONS 423 CITATIONS

SEE PROFILE

\section{Pietro Cipresso}

I.R.C.C.S. Istituto Auxologico Italiano

111 PUBLICATIONS 599 CITATIONS

SEE PROFILE 


\title{
The Social Appearance Anxiety Scale in Italian Adolescent Populations: Construct Validation and Group Discrimination in Community and Clinical Eating Disorders Samples
}

\author{
Antonios Dakanalis ${ }^{1}$ (D) Giuseppe Carrà ${ }^{2} \cdot$ Rachel Calogero $^{3} \cdot$ M. Assunta Zanetti $^{1}$ \\ Chiara Volpato $^{4} \cdot$ Giuseppe Riva $^{5,6} \cdot$ Massimo Clerici $^{7} \cdot$ Pietro Cipresso $^{6}$
}

(C) Springer Science+Business Media New York 2015

\begin{abstract}
Anxiety in situations where one's overall appearance (including body shape) may be negatively evaluated is hypothesized to play a central role in Eating Disorders (EDs) and in their co-occurrence with Social Anxiety Disorder (SAD). Three studies were conducted among community $(N=1995)$ and clinical $(N=703)$ ED samples of 11- to 18-year-old Italian girls and boys to (a) evaluate the psychometric qualities and measurement equivalence/invariance (ME/I) of the Social Appearance Anxiety (SAA) Scale (SAAS) and (b) determine to what extent SAA or other situational domains of social anxiety related to EDs distinguish adolescents with an ED only from those with SAD. Results upheld the one-factor structure and ME/I of the SAAS across samples, gender, age categories, and diagnostic status (i.e., ED participants with and without comorbid SAD). The SAAS demonstrated high internal consistency and 3-week test-retest reliability. The strength of the inter-relationships between SAAS and measures of body image, teasing about appearance, ED
\end{abstract}

Antonios Dakanalis

antonios.dakanalis@unipv.it

1 Department of Brain and Behavioral Sciences, University of Pavia, P.za Botta 11, 27100 Pavia, Italy

2 Division of Psychiatry, Faculty of Brain Sciences, University College of London, London, UK

3 School of Psychology, University of Kent, Canterbury, UK

4 Department of Psychology, University of Milano-Bicocca, Milan, Italy

5 Department of Psychology, Catholic University, Milan, Italy

6 Applied Technology for Neuro-Psychology Laboratory, IRCCS Istituto Auxologico Italiano, Milan, Italy

7 Department of Surgery and Interdisciplinary Medicine, University of Milano-Bicocca, Milan, Italy symptoms, depression, social anxiety, avoidance, and distress, as well as the ability of SAAS to discriminate community adolescents with high and low levels of ED symptoms and community participants from ED participants provided construct validity evidence. Only SAA strongly differentiated adolescents with any ED from those with comorbid SAD (23.2\%). Latent mean comparisons across all study groups were performed and discussed.

Keywords Social Appearance Anxiety Scale . Psychometrics · Social anxiety · Eating Disorders . Comorbidity

\section{Introduction}

Eating Disorders (EDs), characterized by disturbed and inappropriate patterns of eating, excessive focus on appearance, and body image concerns are often inter-related to higher interpersonal sensitivity and social anxiety [1-8]. A great deal of research [6-14] documented associations between negative body image (i.e., dissatisfaction, concern, and distress related to one's appearance $[14,15])$, abnormal eating behaviours, social anxiety symptoms, or fear that one's social self will be judged negatively (recognised as a cognitive vulnerability for, or core feature of, social anxiety $[6,16])$ in female and male samples with or at risk for EDs. There is also compelling evidence that: (a) Social Anxiety Disorder (SAD) has the highest occurrence of all anxiety disorders in EDs, and is significantly more common in ED patients than in controls [8, 17-19]; (b) individuals with $\mathrm{SAD}$ are more likely to report abnormal eating behaviours than controls, and $20 \%$ of them meet criteria for an ED [17, 20]; (c) 20-42\% of individuals with an ED also meet current criteria for SAD diagnosis $[18,20]$; and 
(d) the life time prevalence of SAD is significantly more common in ED patients (i.e., 23-71.5 \%) than in the general population $(12.1 \%)$ [12, 17, 18].

Although it is currently unclear what accounts for this high level of comorbidity, Pallister and Waller [8] suggest three potential explanations. Social anxiety might be (a) a risk factor for EDs, or (b) secondary to eating pathology, or (c) both disorders might share common vulnerability factors. In line with the last explanation, recent experimental manipulations of social appearance anxiety (i.e., the fear that one will be negatively evaluated because of one's appearance [21]) revealed its unique effects on social anxiety, negative body image, and (over- and under-controlled) food intake [22]. These results are in accordance with current conceptualizations of EDs [23] and vulnerability models for both EDs and SAD [9]. Stress from negative social evaluation regarding one's appearance may actually play a pivotal role as a cause of EDs such that social appearance anxiety (SAA) may explain the high occurrence of EDs and SAD [9, 23].

SAA is conceptualized as a negative social evaluative fear that is distinct from a more general fear of negative evaluation [24] because it focuses specifically on the fear of being judged based on appearance [21]. Most of the available research examining SAA has been focused on social physique anxiety as measured by the Social Physique Anxiety Scale (SPAS) [25]. However, this measure was constructed to assess negative thoughts about one's physique and discomfort during a physique evaluation [25]. It refers specifically to body form and structure (i.e., body fat/weight, muscle tone; e.g., "There are times when I am bothered by thoughts that other people are evaluating my weight or muscular development negatively") without taking into account other aspects of appearance, such as shape of facial features and complexion [24, 25]. Furthermore, there is controversy surrounding its factor structure [26], and recent findings from structural equation modeling analyses on adults' data indicated that it might be better thought of as an indicator of negative body image than as a social anxiety construct [24]. In light of these concerns, and because of society's emphasis on the importance of overall appearance and attractiveness in societal judgments [21, 27], the developers of the SPAS constructed the Social Appearance Anxiety Scale (SAAS) to assess perceived anxiety (e.g., nervous, tension) in situations where one's overall appearance (including, but not limited to, body weight, shape or specific body proportions) may be negatively evaluated by others, in both adolescent and adults [24]. The SAAS items [24] were rationally derived from current social anxiety measures and core diagnostic features of both SAD and EDs as presented in DSM-IV [28], making it particularly relevant for a clinical setting [29].
Research on the psychometric properties of the SAAS among four independent American adult samples (respectively $N=512,853,541$, and 323 ) demonstrated a stable unifactorial structure with excellent internal consistency ( $\alpha \mathrm{s}=.93-.95)$ and high 4-week test-retest reliability ( $n=100, r=.84$ ) as well as good construct validity [21, 24]. Specifically the SAAS score was (a) positively associated with measures of social anxiety, negative body image, negative affect, neuroticism, trait anxiety, and depression, (b) negatively related to extraversion, and (c) unrelated to sympathy, openness, agreeableness, conscientiousness, body mass index (BMI), and measures of social desirability response bias. However, SAA does not appear to represent substantial overlap among these constructs. Instead, the SAA has been found to be a unique construct highly related to social anxiety that taps into a unique proportion of variability in social anxiety beyond negative body image, trait anxiety, depression, personality, and negative affect (i.e., SAAS score predicted social anxiety when all of the mentioned constructs were included in the regression equation) [21,24]. Overall the results of the validation studies on adults suggest that the SAAS, in contrast to the SPAS that was only moderately related to the SAAS $(r=.48-.49)$, falls within the larger class of social anxiety [21, 24]. Employing a female adult sample of 60 outpatients with EDs, Claes et al. [10] supported the one-factor model of the SAAS and found that its score was significantly positive in relation to mistrust of others, social detachment, emotional lability and desire to become thinner. Evidence that ED patients obtained higher SAAS scores than matched healthy controls exists as well [29, 30].

Negative interpersonal evaluation involving one's appearance seems as one of the main sources of distress during adolescence $[25-27,31,32]$ in part due to significant physical changes, increased importance of social conformity and desire to appear attractive to members of the opposite sex, and heightened self-consciousness and concerns for peer evaluations [11, 27, 31-35]. However, little is currently known regarding its effects on an adolescent's emotional experiences, well-being and health-related outcomes. Although the construct of SAA is well suited to address this need [24], the examination of its psychometric properties has relied exclusively on adult samples $[10,21,24]$. In order to improve SAAS development, it should be assessed using samples other than adult populations, because measures used with adults cannot be assumed to be appropriate for youngsters who are still developing [16, 34]. Ensuring that SAAS is a reliable and valid tool for assessing adolescent's SAA would guide future research on examining its potential antecedents and effects on adolescent's mental health. For instance, since the onset of eating and body-related symptoms during 
adolescence is linked to an increased risk for physical and mental health problems during adulthood [1,36], studying SAA among adolescents is particularly important to promote future psychological and physical well-being. Studying SAA among adolescents may also provide useful insights in the field of social anxiety, given that (a) adolescence is marked by increases in social anxiety, distress and avoidance and is considered as the time of onset for SAD [16, 34, 37], (b) appearance-focused concerns are included as one of the dimensions along the SAD spectrum [38], and (c) SAA has been suggested to be one of the core concerns causing individuals with SAD to fear public criticism [21].

The available investigations of the psychometric properties of the SAAS in both non-clinical and ED samples $[10,21,24]$ suggest further gaps in the existing literature. Specifically, despite the role that SAA may play in the aetiology of clinical and sub-clinical EDs [11, 22], none of the SAAS validation studies with non-clinical samples [21, 24] examined the association of the SAAS with disordered eating behaviours and its capacity to discriminate individuals with low and high (i.e., at risk for EDs) levels of ED symptomatology. Likewise, while the inter-relationships between general fear of negative evaluation, social avoidance and distress are well documented [34, 37], the degree to which SAA as a specific negative social evaluative fear is associated not only with social avoidance and distress levels, but also with other relevant constructs (i.e., teasing about appearance) [21, 39] remains unknown. Regarding construct validity, the associations between SAA and appearance-related feedback (teasing) may be of particular interest, given that (a) SAAS was developed as a measure that taps into both social anxiety and negative body image constructs [24], and (b) teasing is theorized as a potential antecedent of SAA [24, 39], and was found to be strongly related to social anxiety, body dissatisfaction and distress related to one's appearance [32, 40]. Further, from a statistical standpoint, the size of the ED sample $(N=60)$ in Claes et al.'s [10] study was not appropriate for conducting confirmatory factor analyses (CFA) as it did not exceed the minimum recommended 5:1 cases to parameter ratio (in this case, 16 item-factor parameters and 16 standard error-item parameters) needed to accurately estimate the factor structure of an instrument or a model [41]. Additionally, despite the fact that the sample comprised only women, the association between the SAAS, depression (known to be associated with both SAD and EDs [7]), and other types of social fear (i.e., fear of scrutiny, of negative evaluation, and of social interactions, which represent well-known situational domains of social anxiety [24] and often occur concurrently [6, 21, 24]) were not examined so far. Furthermore, information about the co-occurrence with SAD was not obtained, which might be important for evaluating and understanding whether SAA distinguish ED patients with and without comorbid SAD [9].

It should also be noted that since the SAAS was developed and validated in the United States [21, 24], its translation into another language and subsequent examination of its psychometric proprieties across non English-speaking countries that share the same individualist cultural orientation (e.g., Italy) and those with a collectivist cultural orientation (e.g., Sweden, Estonia, Turkey) [42, 43], would offer the opportunity to provide information regarding the replicability of (or variations in) the factorial structure of the tool and the relationships between the underlining (i.e., SAA) and other theoretical relevant constructs (i.e., behaviour engagement) [24, 42, 44-46]. Finally, prior studies have examined the differences in SAAS observed scores between EDs and healthy controls [29, 30] without establishing and testing the measurement equivalence/invariance (ME/I) of the SAAS. This approach is problematic [41] because if one does not verify that the items of an instrument hold the same meaning and the underlying constructs are perceived similarly in all populations of interest prior to making comparisons across groups, one can never be sure that the reported differences are indicative of true group differences [45]. In fact, if ME/ I, which can be verified via multiple-group CFA [45], is not upheld the validity of the inferences derived from the between-group comparison could be erroneous [41], and inaccurate practical inferences may result [45]. Similarly, assessing ME/I between ED patients with and without comorbid SAD is equally important because there is strong but incomplete comorbidity between SAD and EDs (i.e., not every individual with an ED has SAD) [8]. While reviews [34] seem to indicate that adolescent girls tend to report significantly more social anxiety than adolescent boys, especially with respect to fear of negative evaluation, neither gender differences on the specific fear of being judged based on appearance nor the ME/I of the SAAS across gender has been established. It remains also unknown whether the measurement of SAA is invariant across age categories (i.e., younger vs. older adolescents).

In this manuscript, we present three studies that aim to fill the gaps mentioned above and extend prior research. Study 1 was designed to test the psychometric properties of the Italian version of the SAAS in a large adolescent community sample of both genders. In addition to evaluate its factor structure, internal consistency, and stability over a 3-week period, this study aimed to verify SAAS convergent validity via its relationships not only with body dissatisfaction [21, 24], but also with other unexamined and theoretically relevant constructs (frequency of teasing about appearance, social avoidance and distress, and disordered eating attitudes and behaviours). A further aim of 
Study 1 was to examine, for the first time, the capacity of the SAAS to discriminate participants with high and low levels of ED symptomatology (discriminant validity).

Study 2 had two primary aims. The first was to evaluate whether the factor solution of the SAAS identified in the community adolescent sample (study 1) would be upheld in a large ED treatment seeking sample of adolescents girls and boys (with and without comorbid SAD), and provide further convergent validity evidence (i.e., examination of the associations between SAAS, social fears, depression, and overall severity of ED psychopathology). The second was to detect whether SAA and the other known domains of social anxiety (i.e., fear of scrutiny, fear of negative evaluation, social interaction fear) related to eating disturbances [6] distinguish participants with an ED only from those with an ED and comorbid SAD, and which of these domains produce the largest and most consistent effects across diagnostic groups.

Finally, Study 3 aimed to test the ME/I of the SAAS, and evaluate latent mean differences across gender, age categories (younger vs. older adolescents), samples (community vs. clinical) and diagnostic status (i.e., ED participants with and without comorbid SAD) using the data from the first two studies. We opted to focus on latent means because these are better indicators of true group differences not being associated with measurement error [41, 44, 45].

\section{Methods}

\section{Participants and Procedures}

\section{Study 1}

Data for this study, collected based on the methodology of Crocetti et al. [47], were drawn from the baseline assessment of a five-wave longitudinal study assessing risk factors for EDs [36]. The participating students consisted of two cohorts of younger (i.e., 11-14 years) (493 girls and 510 boys; $M_{\text {years }}=12.30, S D=0.75 ; \quad M_{B M I}=19.99$, $S D=1.79)$ and older (i.e., 15-18 years) adolescents (502 girls and 490 boys; $M_{\text {years }}=16.01, \quad S D=0.99$; $M_{B M I}=20.58, S D=2.67$ ) attending various junior high and high schools in the Northern, Central, and Southern regions of Italy. ${ }^{1}$ An active parental consent procedure was

\footnotetext{
${ }^{1}$ As the two cohorts respectively reflect students from Italian junior high and high schools [47], in line with prior Italian research [47] examining the psychometric proprieties and ME/I of self-reported measures of anxiety (social anxiety included), the younger (11-14 years) and older (15-18 years) adolescent groups were maintained in the analyses investigating age differences. Self-reported weight and height were used to calculate BMI $\left(=\mathrm{kg} / \mathrm{m}^{2}\right)$.
}

used to recruit participants, ${ }^{2}$ resulting in an average participation rate of $59.5 \%$ of eligible adolescents (participation was voluntary). The participation rate is close to that of other school-based samples (i.e., 58-60\%) using the same active parental consent procedure, without offering incentives [2, 11]. Moreover, the total analytic sample $(N=1995)$, which contained nearly equal proportions of adolescents from urban, suburban, and rural communities from Northern, Central, and Southern Italy [36], was representative in terms of gender $(50.3 \%)$, ethnic composition (93\% Caucasian; $2.2 \%$ Hispanic/Latino; and $4.8 \%$ other or mixed ethnic heritage) and family-socioeconomic status $(66.5 \%$ middle class; $20.1 \%$ lowermiddle class; and $13.4 \%$ upper-middle class) of the total Italian junior high and high school populations. ${ }^{3}$ The majority of the analytic sample (49.9\% females) self-described as Caucasian (93.5 \%), 2 \% identified as Hispanic/ Latino, and $4.5 \%$ specified other or mixed racial heritage. For socio-economic status, $66.5 \%$ of participants' parents reported middle class, $20 \%$ endorsed lower-middle class, and $13.5 \%$ indicated upper-middle class; there were no differences in these socio-demographic characteristics across the two cohorts.

In addition to the Italian version of the SAAS, selected measures (see below) relevant to the goals of this study (and others that were outside the scope of the current analyses [36]) were presented in a counterbalanced order to offset possible order effects. Instructions on how to respond to items in each instrument were provided by professional clinical psychologists, under the supervision of the researchers. A second assessment of the SAAS was conducted 3 weeks later $\left(M_{\text {days }}=21.03, S D=0.89\right.$; range 19-23), in eleven of the classes randomly selected [47]. Thus, the test-retest data set includes 152 younger ( 74 girls and 78 boys; $M_{\text {years }}=12.23, S D=0.71$ ) and 157 older ( 79 girls and 78 boys; $M_{\text {years }}=15.88, S D=1.04$ ) adolescents, who were the same age at both time points; analysis verified that the subset of these participants did not differ significantly on demographic factors or any of the study variables from those who completed the SAAS only during the first administration. The study was approved by the Institutional Review Board of the University of Pavia.

\section{Study 2}

Individuals consecutively referred to and assessed for treatment of an ED at three medium to large size

\footnotetext{
2 Participants' assent was also secured immediately before the assessment, which took place on the school campuses (after consent from school administrators was obtained) [36].

${ }^{3}$ Italian Census Bureau (ISTAT): www.istat.it; further detailed information is also available elsewhere [2, 11, 47].
} 
specialized care centres for child and adolescent EDs (allocated in Northern, Central, and Southern Italy) between January 2010 and January 2014 were asked to participate in the study if the following criteria were met: (a) age 11-18 years, (b) current DSM-IV ${ }^{4}$ [28] diagnosis of Anorexia Nervosa (AN), Bulimia Nervosa (BN), or ED Not Otherwise Specified (EDNOS), (c) fluency in Italian, (d) no mental retardation or pervasive developmental disorders, and (e) no previous evidence of any physical conditions (e.g., diabetes mellitus) known to influence eating or weight. Of 713 adolescents who met study eligibility criteria, 10 subjects did not participate, mainly because the study seemed too time consuming $(n=8)$ or because families had no interest in the study $(n=2)$; non-participants did not differ from participants $(98.6 \%)$ on sociodemographic and clinical measures or any other variables assessed in the study. The final sample (633 girls and 70 boys [9.9\%]) comprised $212(30.2 \%)$ adolescents with AN $\left(M_{B M I}=15.52, S D=1.24\right), 131(18.6 \%)$ with BN $\left(M_{B M I}=21.18, \quad S D=2.07\right)$ and $360 \quad(51.2 \%)$ with EDNOS $\left(M_{B M I}=20.05, S D=3.54\right)$. Two age groups were represented in the entire sample $(N=703)$ : a younger (i.e., 11-14 years) adolescent group (189 girls and 15 boys; $M_{\text {years }}=12.59, S D=0.70$ ) and an older (i.e., 15-18 years) adolescent group (444 girls and 55 boys; $M_{\text {years }}=16.74, S D=0.61$ ). Most of them self-identified as Caucasian $(92 \%)$, while in terms of family-socioeconomic status, $69 \%$ of participants' parents reported middle class; there were no differences between girls and boys or between diagnostic groups and age categories in these socio-demographic characteristics.

The current prevalence of SAD in the entire ED sample and across ED types was estimated. One hundred and sixtythree $(23.2 \%)$ of the adolescents with an ED (49 with AN [23.1\%], 32 with BN [24.1\%], and 82 with EDNOS [22.6\%]; $\chi^{2}(2)=0.91 n s$ ) had current comorbid SAD.

The baseline routine assessment included: (a) the Italian version of the Eating Disorder Examination (EDE)-12.0D [48] for assessing the DSM-IV ED diagnoses, (b) the Italian version of the Kiddie Schedule for Affective Disorders and Schizophrenia-Present version (K-SADS-P) [49] for assessing the presence or absence of current DSMIV SAD, ${ }^{5}$ and (c) measurement of height and weight via calibrated electronic instruments [2, 7], from which BMI $\left(\mathrm{kg} / \mathrm{m}^{2}\right)$ was calculated. Participants also completed

\footnotetext{
4 These criteria were used since the study was initiated before the official publication of the DSM-5.

5 Inter-rater reliability for ED and SAD diagnoses was determined by having two randomly selected samples of $25 \%$ of the EDEs-12.0D $(\kappa=1.0)$ and $25 \%$ of the K-SADS-Ps $(\kappa=1.0)$ that were conducted at each participating site rated by a second blinded clinician (with almost 10-years experience in assessing and treating ED and comorbid disorders among adolescents) at the other site.
}

selective standardized measures (described below) in counterbalanced order in an attempt to offset possible ordering effects. In line with the legal requirements of the study country (Italy), informed written consent was sought from subjects and their legal guardians prior to inclusion in the study after all study procedures were fully explained and before individuals were being triaged to a treatment program. This study was also approved by the ethics review board of each local institution/participating site and the Institutional Review Board of the co-ordinating body for this project (University of Pavia).

\section{Study 3}

This study used the combined data of Studies 1 and 2 .

\section{Measures}

SAAS (Studies 1-2). The 16 items of the SAAS [24] are rated on a 5-point Likert-type scale $(1=$ not at all, $5=$ extremely), with higher scores reflecting higher SAA. All items were initially translated to Italian (SAAS) and then back-translated into English by an independent bilingual researcher with experience in conducting translations for health surveys [50], after permission from the developers of the original (English) SAAS was obtained. The two English versions of the Italian-SAAS were compared and the few inconsistencies highlighted were removed in a further translation and back-translation comparison process until the two versions were identical [50]. The items of the final Italian SAAS (Table 1) did not show any meaningful differences from the original English version, as also confirmed by two independent professional translators [44, 50].

In addition to the 16-item SAAS, ${ }^{6}$ participants in Studies 1 and 2 were given the Italian version of the measures described below (their estimated Cronbach's alphas are given in Table 2), which have demonstrated good construct validity, high internal consistency and test-retest reliability amongst Italian community samples and a variety of

\footnotetext{
${ }^{6}$ As in prior research [26] a preliminary independent study was conducted to evaluate the content clarity of the Italian SAAS. Ninety adolescents ( $49 \%$ boys; $M_{\text {years }}=11.22, S D=0.35$, range $11-12$ ) recruited from two schools (from Northern and Southern Italy) completed the Italian SAAS with its original response format replaced by a 5 -point Likert scale assessing the clarity of the items $(1=$ not at all clear, $5=$ completely clear $)$. Analyses of the clarity of the items were performed following Vallerand's [52] suggestions, including the recommendation that an item clarity score $<4$ out of five should be considered unsatisfactory on a 5-point scale. We considered all items satisfactory, with observed scores ranging from a low of 4.34 $(S D=0.25)$ for Item 12 through a high of $4.77(\mathrm{SD}=0.11)$ for Item 3 . For interested readers the item-specific results are available from the corresponding author on request.
} 
Table 1 Social Appearance Anxiety Scale (SAAS) items and factor loadings obtained from Studies 1 and 2

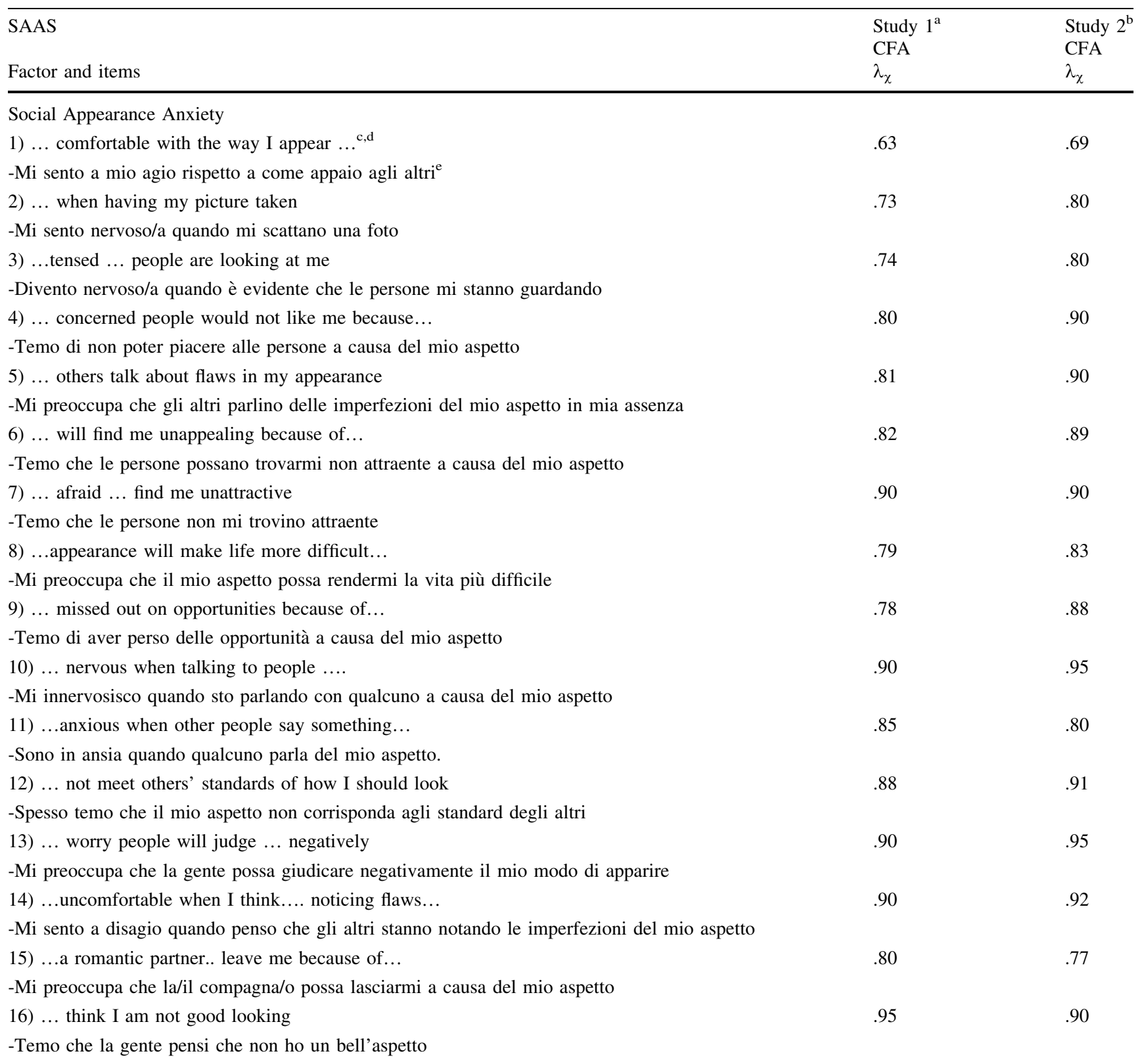

CFA confirmatory factor analysis, $\lambda_{\chi}$ standardized coefficients

a 1995 adolescents from community ( $n=995$ girls, $n=1000$ boys)

b 703 adolescents with Eating Disorders ( $n=633$ girls, $n=70$ boys)

${ }^{c}$ Exact wording for SAAS items cannot be shown due to copyright restrictions for the original (English) version of the SAAS. () 2008 Sage Publications

d Item 1 is Reverse-coded

e Italian translation of the SAAS. Permission to use this measure is not required. However, we do request that you notify the corresponding author via e-mail if you use the Italian SAAS in your research. Please seek permission if any item is modified

psychiatric samples of adults and adolescents ( $\geq 11$ years) of both genders. The references provided contain detailed information about the psychometric qualities of the Italian measures used in these studies.

\section{Social Avoidance and Distress Scale (SADS; Study 1)}

The Italian SADS [51] is a 28-item true-false scale grouped into two subscales: "social avoidance" (14 items) 
Table 2 Descriptive statistics, internal consistency values, and correlations with the Social Appearance Anxiety Scale for younger and older adolescent girls and boys across Studies 1-2

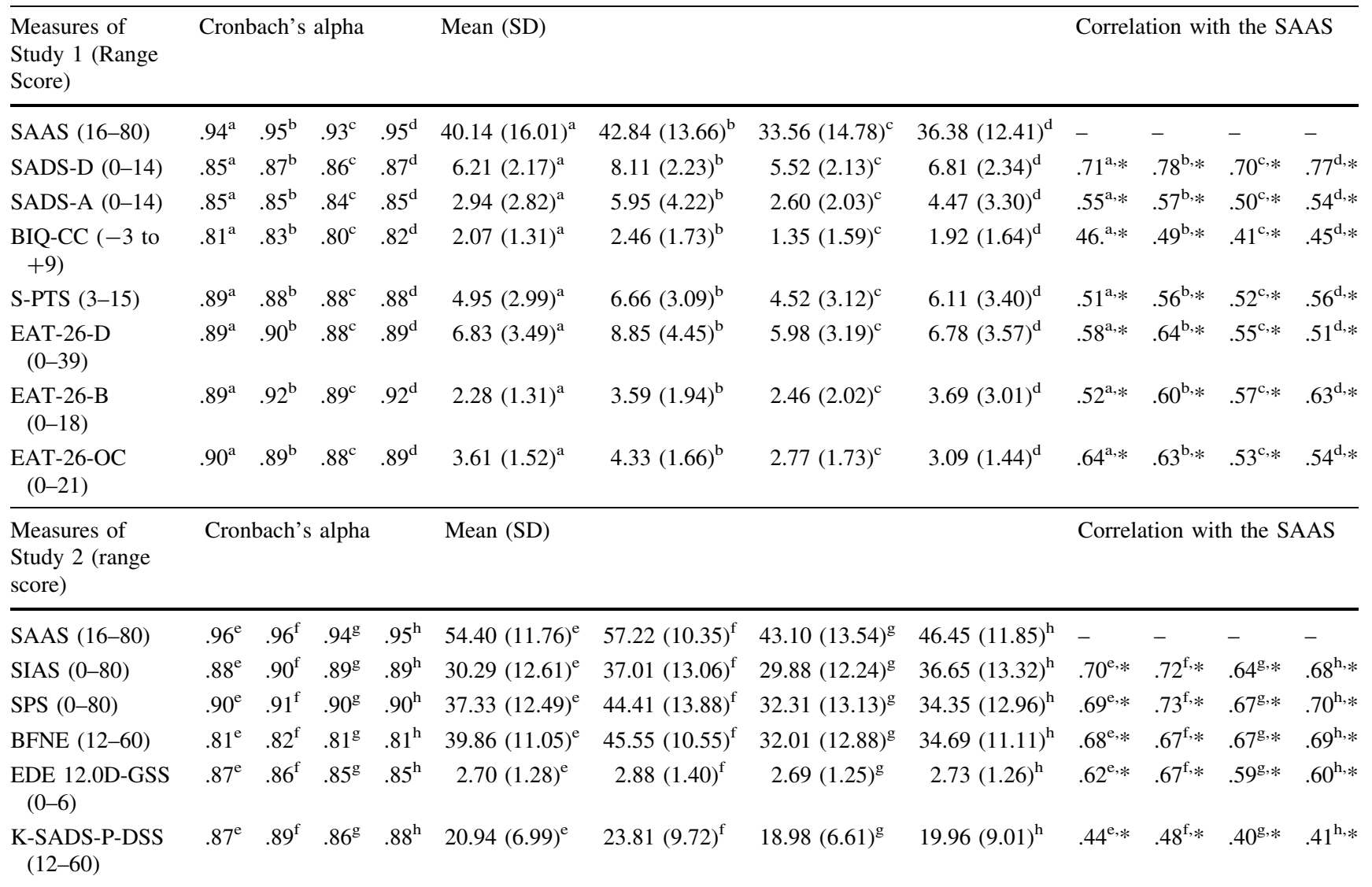

SAAS Social Appearance Anxiety Scale, SADS-D and SADS-A Distress and Avoidance subscales of the Social Avoidance and Distress Scale, $B I Q-C C$ Body-Image Ideals Questionnaire-Composite Score, S-PTS Short-Perception of Teasing Scale, EAT-26-D, B, and OC Dieting, Bulimia, and Oral Control sub-scales of the Eating Attitudes Test-26, SIAS Social Interaction Anxiety Scale, SPS Social Phobia Scale, BFNE Brief Fear of Negative Evaluation Scale, EDE 12.0D-GSS Global severity score of the Eating Disorder Examination Interview 12.0D, K-SADS-P-DSS Symptom composite score from the depressive symptom section of the Kiddie Schedule for Affective Disorders and Schizophrenia InterviewPresent version

$* p<.001$

a Younger (11-14 years) adolescent girls from community $(n=493)$

b Older (15-18 years) adolescent girls from community $(n=502)$

c Younger (11-14 years) adolescent boys from community $(n=510)$

${ }^{\mathrm{d}}$ Older (15-18 years) adolescent boys from community $(n=490)$

e Younger (11-14 years) adolescent girls with Eating Disorders $(n=189)$

${ }^{\mathrm{f}}$ Older (15-18 years) adolescent girls with Eating Disorders $(n=444)$

g Younger (11-14 years) adolescent boys with Eating Disorders $(n=15)$

${ }^{\text {h }}$ Older (15-18 years) adolescent boys with Eating Disorders $(n=55)$

and "distress" (14 items), respectively assessing the tendency to avoid social situations [e.g., "If the chance comes to meet new people (e.g., parties), I often take it"; reverse item] and social inhibition, distress, and discomfort in social situations or with unfamiliar people (e.g., "It is easy for me to relax when I am with strangers"; reverse item). Subscale scores range from 0 (lowest distress and avoidance) to 14 (highest distress and avoidance).
Short-Perception of Teasing Scale (S-PTS; Study 1)

The Italian S-PTS [53] was used to assess the frequency of negative appearance-related feedback (teasing) from significant others (i.e., parents, friends, peers). The three items (e.g., "Your friends tease you about your appearance") are rated on a 5 -point Likert $(1=$ never, $5=$ always $)$; higher scores indicate more frequent appearance-related teasing. 
Body-Image Ideals Questionnaire (BIQ; Study 1)

The BIQ is typical used in research as a measure of body dissatisfaction [32, 54]. For 11 physical attributes (e.g., weight, shape, muscle tone and definition, body proportions, overall physical appearance), two different sets of ratings are assessed. For the discrepancy ratings, participants indicate on a 4-point scale how much they think they resemble their personal ideal of that physical attribute $(0=$ exactly as $I$ am, $3=$ very unlike me $)$. For the importance ratings, participants indicate on a 4-point scale how important it is that they embody that physical ideal $(0=$ not important, $3=$ very important $)$. In the current study, the Italian version of the BIQ was used [36]. Consistent with prior research [54], a composite score is calculated as the mean of the item-by-item product of discrepancy and importance ratings, after the discrepancy scores of 0 are recoded to a value of -1 to allow for self-congruent items (exactly as $I \mathrm{am}$ ) to be weighted by importance. A higher score indicates a greater disparity between perceived, actual, and ideal physical attributes, with associated (greater) emphasis on meeting physical ideals.

\section{Eating Attitudes Test-26 (EAT-26; Study 1)}

The Italian EAT-26 [55] assesses symptoms and characteristics of eating disturbances and has been validated as both a both dimensional and categorical measure. The 26 items form three subscales: "dieting" (13 items) "bulimia" (6 items) and "oral control" (7 items), respectively assessing avoidance of fatty foods and preoccupation with losing weight, bulimic tendencies, and food intake mode and its control. Participants were asked to indicate how frequently each item characterizes them (e.g., "Particularly avoid food with a high carbohydrate content: bread, rice, potatoes, etc."; "Cut my food into small pieces") on a 6-point scale (ranging from never to always). Consistent with EAT-26 scoring criteria [55], the choices always, usually, and often were recoded as 3, 2, and 1 , whereas the remaining three choices were recoded to 0 . In addition to the subscale scores (a higher score reflects a greater manifestation of the particular construct measured), all items were totalled to obtain a global scale score; the cut-off point for considering an individual at risk for EDs is a score of 20 or above [55]. In the community sample, $5.1 \%(n=25)$ and $10.3 \%(n=52)$ of younger and older adolescent girls, and $1.9 \%(n=10)$ and $2.6 \%(n=13)$ of younger and older adolescent boys scored $\geq 20$; these percentages are quite close to those found by previous Italian community-based studies on junior high (11-14 years) and high school (15-18 years) students $[55,56]$.
Social Interaction Anxiety Scale and Social Phobia Scale (SIAS; SPS; Study 2)

The Italian SIAS and SPS [57] were used to assess fear of interacting in dyads and groups (e.g., "I have difficulty talking with other people") and fear of being scrutinized by others during routine activities, such as eating, drinking, or writing (e.g., "I become anxious if I have to write in front of other people"), respectively. Each scale contains 20 items rated on a Likert-type scale $(0=n o t$ at all; $4=$ extremely), with a higher score indicating greater social interaction anxiety and fear of scrutiny, respectively.

Brief Fear of Negative Evaluation Scale (BFNE; Study 2)

The Italian BFNE [51] was used to assess fear of negative evaluation in social situations, which is a core component of social anxiety [16]. The 12 items (e.g., "I am afraid others will not approve of me") are rated on a Likert-type scale $(1=$ not at all, $5=$ extremely); higher scores indicate greater fear.

\section{EDE-12.0D (Study 2)}

Except for diagnostic items, the Italian EDE-12.0D [48] yields four subscales (restraint, shape concern, weight concern, and eating concern), and a global score measuring the overall severity of ED psychopathology over the previous 4 weeks. In this work, the global score was used, which ranges from 0 to 6 , with higher scores reflecting greater overall severity $[7,48]$.

\section{K-SADS-P (Study 2)}

Depressive symptoms were assessed using the depressive symptom Sect. (12 items) of the Italian K-SADS-P interview (K-SADS-P-DSS) [49]. Symptoms are rated on a 6-point scale (0-5) with severity ratings ranging from none to severe. Severity ratings for each symptom were averaged to form a symptom composite, which has been shown to be a reliable measure of depressive symptom severity [58].

\section{Data Analytic Plan}

\section{Study 1}

A total of $9.5 \%(n=190)$ of participants had at least one missing data point (the count for missing data points ranged from 0 to $0.85 \%$ ). Multiple imputation in SPSS 19.0 [59] was used to estimate missing data points by creating five imputed data sets using available data [because they were missing completely at random (Little's Missing Completely at Random test: $\left.\chi^{2}(377)=394.95, p=.27\right)$ ], 
and subsequent analyses were conducted on all five data sets, with pooled results. We examined the factor structure of the Italian SAAS by means of CFA, to check whether its underlying latent structure corresponded to the original (English) version (Model 0a). Based on the results of earlier psychometric studies of the SAAS [21, 24], (a) a one factor model with 16 SAAS items indicating SAA was specified, and (b) errors terms between certain SAAS items were allowed to correlate due to similarity in wording [41]. Specifically, four items that begin with "I worry that..." (n. 5, 8, 13, and 15); four items that begin with "I am concerned people..." (n. 4, 6, 9, and 16); and two items that refer to feeling "nervous" (n. 2 and 10). The CFA was performed in Mplus 6.12 [60] using the maximum likelihood (ML) estimator. ${ }^{7}$ Comparative fit index (CFI) and Tucker-Lewis incremental fit (TLI) index values $\geq .95$, standardized root mean square residual (SRMR) values $\leq .08$, and standardized root mean square residual (RMSEA) values $\leq .06$ were used as standards for good model fit [63]. Despite Chi square statistic's $\left(\chi^{2}\right)$ dependency and sensitivity on sample size [41], the $\chi^{2}$ values are reported as well.

Internal consistency reliability of the SAAS was assessed using Cronbach's alpha coefficient ( $\alpha$ values of 70, .80 and .90 or above are considered satisfactory, good and excellent, respectively) [64], and intraclass correlation coefficients (ICCs) were used to estimate the test-retest reliability of the SAAS using data from the subsets of adolescents who completed the SAAS at both administrations 3 weeks apart [64]. To determine the convergent validity between the SAAS the other measures of Study 1, we made use of Pearson correlations (adjusted $p$ value $=.006$ ); Cohen's [65] criteria $(r s)$ were used to determine the strength of the associations (slight $\geq .10$, medium $\geq .30$, large $\geq .50$ ). Concerning discriminant validity, independent sample $t$ tests were performed to examine differences in scores on the SAAS between adolescents with elevated $(\geq 20)$ and non-elevated (global) scores on the EAT-26 [55]. Effect sizes as measured by Cohen's [65] $d$ were also reported $($ small $=.20$, medium $=.50$, large $=.80$ ). The above analyses were performed in SPSS

\footnotetext{
7 ML estimator treats measured responses as continuous [41]. According to simulation studies, when variables/items are measured on an ordinal scale and contain five (or more) categories (such as SAAS items rated on a 5-point Likert-type scale), these could be safely treated as continuous, if they are not skewed or kurtotic [61]. The results of pre-analyses of data of all studies reported in the current manuscript indicated that skewness, kurtosis and Mardia's normalized values for all SAAS items were well below critical limits, i.e., skewness $<|2.0|$; kurtosis $<|7.0|$; and Mardia's normalized values $<3$ [41]. Hence, the use of ML estimator was deemed appropriate and it is also consistent with prior research, based on similar assumptions, and using the same estimator for examining the factor structure of a social anxiety measure matching the format response of I-SAAS [62].
}

19.0 [59] and the results obtained in each subsample of younger and older adolescent girls and boys were reported.

Study 2

There were no missing data in this study. To evaluate whether the factor structure of the SAAS that was identified among community adolescents (Study 1) is upheld in sample of adolescents with EDs, we performed a CFA in Mplus 6.12 [60] with ML estimator (see footnote 7) (Model 0b), and applied the same guidelines for conducting CFA as outlined in Study 1. As in the first study, we calculated the internal consistency of the SAAS and its correlations (adjusted $p$ value $=.008)$ with the other measures of the study (convergent validity) for each subsample of younger and older adolescent girls and boys, using SPSS 19.0 [59]. To identify to what extent SAA and other domains of social anxiety (i.e., fear of scrutiny, of social interactions, and of negative evaluation) distinguish individuals with only an ED (i.e., AN, BN or EDNOS) from those with an ED and $\mathrm{SAD},{ }^{8}$ and which of these domains produce the largest and most consistent effects across groups, logistic regression analyses were conducted. Following Allison's [66] guideline, regressions performed in SAS 9.3 [67] were stratified by ED diagnosis, and controlled for depression, gender, BMI, and age [7, 9, 21]. Variables entered simultaneously ${ }^{9}$ into logistic regression models were standardized by the use of Proc Standard to make interpretations of odds ratios for independent variables more comparable; odds ratios whose confidence limits exclude 1 are statistically significant [66]. To further assess the impact of variables, effect sizes (Cohen's $d$; see Study 1) were calculated [66].

\section{Study 3}

We tested the ME/I of the SAAS and latent mean differences across groups [i.e., adolescents from community versus adolescents with EDs, girls versus boys (including clinical and non-clinical participants) [62], younger versus older adolescents (including clinical and non-clinical participants) [62], and ED participants with comorbid SAD versus ED participants without $\mathrm{SAD}$ ] within a multi-group

\footnotetext{
${ }^{8}$ Given the dearth of research regarding domains of social anxiety as potential differentiators across EDs and specific ED diagnoses, the three ED diagnostic groups (AN, BN, EDNOS) were examined separately for comparative reasons.

${ }^{9}$ Given that in pre-analyses all variables examined separately (controlled for gender, BMI, age and depression) showed a significant difference between groups $(p<.01)$, we entered all variables simultaneously into logistic regression models to determine the factor(s) that best distinguished individuals who reported an ED + SAD compared to those who only reported an ED [66]. The results of pre-analyses are available from the corresponding author on request.
} 
CFA framework [41], using the combined data from Studies 1 and 2. Before conducting an ME/I test (multigroup CFA), we assessed the model fit (i.e., single CFA) for each group separately (Models $0 \mathrm{c}-\mathrm{h}$ ) ${ }^{10}$ to ensure adequate factorial validity of the 16-item model, as recommended [41, 45]; the guidelines for conducting CFA outlined in Study 1 was applied.

Byrne [41] presented a hierarchical sequence of models for establishing ME/I that were used in the current investigation. Model 1 examined "configural invariance," the basic level of invariance between each pair of groups, in which the only invariance constraint was that the same parameters exist across groups [41]. If it is met, it only suggests that the factor structure is equivalent [45] across groups (in this case, the 1-factor structure best represents the data for all). Model 2 tested for "metric" (or factor loading) invariance by containing the restrictions of Model 1, in addition to equal constraints on factor loadings between groups. Establishing this type of invariance indicates that different groups (i.e., ED patients with and without comorbid SAD) perceive, interpret, and respond to the items in a similar manner [41]. Model 3 tested for "scalar" (or intercept) invariance, which included the restrictions of Model 2, in addition to equal constraints on item intercepts. This model explores whether individuals with the same value on the latent construct would have equal values on the observed variable [45] and is a requirement for the comparison of latent means across groups [41]. ${ }^{11}$ The analyses were performed in Mplus 6.12 [60], using ML estimation (see footnote 7) on the covariance matrix and mean vector (MACS); for more information and the advantages of the MACS analysis, see Byrne [41]. Because of the $\chi^{2}$ test's dependency and sensitivity on sample size and model complexity, which may result in significant changes in fit even when such changes are diminutive [41, 68], we presented the $\chi^{2}$ values (Table 3 ), but we did not interpret them as indicators of fit, in line with prior research $[44,62]$. In order to evaluate the degree of ME/I, the invariance models (Models 1-3) were assessed comparatively [41] by examining the changes $(\Delta)$ in CFI, RMSEA, and SRMR [57]; if $\Delta$ CFI $\leq .010, \quad \Delta$ RMSEA $\leq .015$, and $\triangle \mathrm{SRMR} \leq .030$ for tests of factor loading invariance, and $\Delta \mathrm{CFI} \leq .010, \Delta \mathrm{RMSEA} \leq .015$, and $\Delta \mathrm{SRMR} \leq .010$ for tests of intercept invariance, then ME/I is demonstrated [68].

\footnotetext{
${ }^{10}$ Except for data collected from adolescents recruited from the community (Model 0a) and adolescents with EDs (Model 0b) that have been independently analysed in the first two studies (see data analytic plan and results sections).

11 Although the equality of the uniqueness matrix can be assessed as part of the ME/I [45], in line with prior research [44, 62] we did not analyse this characteristic, since the equivalence of error matrices is not necessary when the observed scores are used merely as indicators of latent variables [41], and as noted we focused on the latent variables.
}

Latent factor mean differences across groups were performed by fixing the latent mean values to zero for one group (which operates as a "reference" group) against which the freely estimated latent mean values for the other group is compared [41]. Statistical significance associated with differences between the latent means was determined on the basis of the z-statistic [41, 44, 45]. The effect size (Cohen's $d$; see Study 1) associated with the latent mean differences was estimated according to the guidelines of Byrne [41].

\section{Results}

\section{Study 1: Community Sample}

The adequacy of the one-factor structure of the SAAS was examined using CFA. As shown in Table 3, the one-factor model provided a good fit to the data (Model 0a). ${ }^{12}$ All indicators (items) loaded significantly on the SAA latent factor; these standardized estimates are given in Table 1. Thus, the original single-factor structure of the SAAS is applicable in Italian community adolescents.

Table 2 reports the descriptive statistics and Cronbach's alpha coefficients for the SAAS from the sub-sample of younger and older adolescent girls and boys. The SAAS demonstrated excellent internal consistency [64] across gender and age groups. Using data from a subset of 309 adolescents who completed the SAAS at both administrations 3 weeks apart, we estimated high test-retest reliability [64]: the association between the first and second administration (ICC) was .83 for younger adolescent girls $(n=74)$ and .81 for boys $(n=78)$, and .81 for older adolescent girls $(n=79)$ and .84 for boys $(n=78)$. Convergent validity was provided by the moderate correlations between the SAAS and BIQ composite scores, and the strong positive associations between the SAAS and teasing about appearance, social avoidance, distress, and the three EAT-26 subscales (dieting, bulimia, and oral control) noted across all age and gender subsamples (see Table 2). Support for the discriminant validity of the SAAS was observed from the results of the independent sample $t$ tests, which revealed differences in SAAS scores between the younger and older adolescent girls and boys with elevated $(\geq 20)$ and non-elevated (global) scores on the EAT-26 [55]. Specifically, scores on the SAAS were significantly higher in younger $(M=42.11, S D=13.54)$ and older adolescent girls $(M=48.39, S D=10.81)$ with elevated EAT-26 scores than in those with lower scores

\footnotetext{
12 Modification indices provided by Mplus [60] were detected in this study as well as in the following studies reported, but in all cases their magnitude $(<5.0)$ suggested that any not originally specified parameters did not impact the fit of model to the data [41].
} 
Table 3 Social Appearance Anxiety Scale (SAAS): goodness-of-fit indices of the one-factor model and measurement equivalence/invariance across groups

\begin{tabular}{|c|c|c|c|c|c|c|c|c|c|c|c|}
\hline 16-Item Model & $\chi^{2}$ & $\Delta \chi^{2}$ & $d f$ & $\mathrm{CFI}$ & TLI & SRMR & RMSEA & Comparison & $\Delta \mathrm{CFI}$ & $\Delta \mathrm{SRMR}$ & $\triangle \mathrm{RMESA}$ \\
\hline \multicolumn{12}{|l|}{ Single CFAs } \\
\hline $\begin{array}{l}\text { Model 0a: Adolescents from } \\
\text { community-Study } 1(N=1995)\end{array}$ & $264.03 *$ & & 91 & .979 & .978 & .041 & .046 & & & & \\
\hline $\begin{array}{l}\text { Model 0b: Adolescents with EDs - } \\
\text { Study } 2(N=703)\end{array}$ & $202.94 *$ & & 91 & .989 & .988 & .032 & .036 & & & & \\
\hline Model 0c: Girls ${ }^{\mathrm{a}}-(N=1628)$ & $232.05^{*}$ & & 91 & .985 & .984 & .034 & .040 & & & & \\
\hline Model Od: $\operatorname{Boys}^{\mathrm{a}}(N=1070)$ & $244.77 *$ & & 91 & .983 & .982 & .036 & .042 & & & & \\
\hline $\begin{array}{l}\text { Model 0e: Younger Adolescents }{ }^{\mathrm{a}, \mathrm{b}} \\
(N=1207)\end{array}$ & $253.99 *$ & & 91 & .981 & .981 & .038 & .044 & & & & \\
\hline $\begin{array}{l}\text { Model 0f: Older Adolescents }{ }^{\mathrm{a}, \mathrm{c}} \\
(N=1491)\end{array}$ & $224.87 *$ & & 91 & .986 & .985 & .034 & .039 & & & & \\
\hline $\begin{array}{l}\text { Model 0g: ED participants with } \\
\text { comorbid } \operatorname{SAD}(N=163)\end{array}$ & $194.91 *$ & & 91 & .990 & .990 & .030 & .035 & & & & \\
\hline $\begin{array}{l}\text { Model Oh: ED participants without } \\
\text { comorbid SAD }(N=540)\end{array}$ & $205.55^{*}$ & & 91 & .988 & .987 & .033 & .038 & & & & \\
\hline \multicolumn{12}{|l|}{ Test for ME/I: Multiple-group CFAs } \\
\hline \multicolumn{12}{|l|}{$\begin{array}{l}\text { Adolescents from community versus } \\
\text { Adolescent with EDs }\end{array}$} \\
\hline $\begin{array}{l}\text { Model 1: Configural Invariance } \\
\text { Model }\end{array}$ & $466.97 *$ & & 182 & .984 & .983 & .037 & .041 & & & & \\
\hline $\begin{array}{l}\text { Model 2: Factor Loading Invariance } \\
\text { Model }\end{array}$ & $504.34 *$ & $37.37 *$ & 197 & .981 & .981 & .040 & .043 & 2 versus 1 & .003 & .003 & .002 \\
\hline \multicolumn{12}{|l|}{ Girls versus Boys ${ }^{\mathrm{a}}$} \\
\hline $\begin{array}{l}\text { Model 1: Configural Invariance } \\
\text { Model }\end{array}$ & $476.82 *$ & & 182 & .984 & .983 & .035 & .040 & & & & \\
\hline $\begin{array}{l}\text { Model 2: Factor Loading Invariance } \\
\text { Model }\end{array}$ & $506.93 *$ & $30.11 *$ & 197 & .983 & .982 & .036 & .041 & 2 versus 1 & .001 & .001 & .001 \\
\hline \multicolumn{12}{|l|}{$\begin{array}{l}\text { Younger }^{\mathrm{a}, \mathrm{b}} \text { versus Older } \\
\text { Adolescents }^{\mathrm{a}, \mathrm{c}}\end{array}$} \\
\hline $\begin{array}{l}\text { Model 1: Configural Invariance } \\
\text { Model }\end{array}$ & $478.86^{*}$ & & 182 & .983 & .983 & .036 & .042 & & & & \\
\hline $\begin{array}{l}\text { Model 2: Factor Loading Invariance } \\
\text { Model }\end{array}$ & $512.18^{*}$ & $33.32 *$ & 197 & .981 & .980 & .038 & .044 & 2 versus 1 & .002 & .002 & .002 \\
\hline Model 3: Intercept Invariance Model & $581.07 *$ & $68.89 *$ & 212 & .979 & .978 & .040 & .046 & 3 versus 2 & .002 & .002 & .002 \\
\hline \multicolumn{12}{|l|}{$\begin{array}{l}\text { ED participants with SAD versus ED } \\
\text { participants without comorbid SAD }\end{array}$} \\
\hline $\begin{array}{l}\text { Model 1: Configural Invariance } \\
\text { Model }\end{array}$ & $400.46^{*}$ & & 182 & .989 & .989 & .032 & .036 & & & & \\
\hline $\begin{array}{l}\text { Model 2: Factor Loading Invariance } \\
\text { Model }\end{array}$ & $430.31 *$ & $29.85^{*}$ & 197 & .988 & .988 & .033 & .037 & 2 versus 1 & .001 & .001 & .001 \\
\hline Model 3: Intercept Invariance Model & $490.97 *$ & $60.66^{*}$ & 212 & .987 & .987 & .034 & .038 & 3 versus 2 & .001 & .001 & .001 \\
\hline
\end{tabular}

$\chi^{2}$ Chi square test, $\Delta$ difference values, $d f$ degree of freedoms, CFI comparative fit index, TLI Tucker-Lewis Incremental fit index, SRMR standardized root-mean square residual, RMSEA root-mean square error of approximation, CFAs Confirmatory factor analyses, EDs Eating Disorders, $S A D$ Social Anxiety Disorder, $M E / I$ measurement equivalence/invariance

$* p<.001$

${ }^{a}$ Including clinical and non-clinical participants

b $11-14$ years

c $15-18$ years 
Table 4 Odds ratios, $99 \%$ confidence intervals, and effect sizes (d) from logistic regression analyses

\begin{tabular}{|c|c|c|c|c|c|c|}
\hline Variable (instrument) & $\begin{array}{l}\text { AN + SAD versus } \\
\text { AN only }\end{array}$ & $d$ & $\begin{array}{l}\mathrm{BN}+\mathrm{SAD} \text { versus } \\
\mathrm{BN} \text { only }^{\mathrm{a}}\end{array}$ & $d$ & $\begin{array}{l}\text { EDNOS + SAD versus } \\
\text { EDNOS only }\end{array}$ & $d$ \\
\hline $\begin{array}{l}\text { Social Appearance Anxiety (Social Appearance } \\
\text { Anxiety Scale) }\end{array}$ & $3.01(2.35,3.44)^{*}$ & .89 & $3.25(2.75,3.73)^{*}$ & .96 & $3.13(2.28,3.52)^{*}$ & .92 \\
\hline $\begin{array}{l}\text { Fear of Social Interactions (Social Interaction } \\
\text { Anxiety Scale) }\end{array}$ & $0.97(0.72,1.26)$ & .02 & $1.16(0.93,1.44)$ & .12 & $0.98(0.79,1.38)$ & .03 \\
\hline $\begin{array}{l}\text { Fear of Negative Evaluation (Brief Fear of } \\
\text { Negative Evaluation Scale) }\end{array}$ & $1.00(0.86,1.14)$ & .00 & $1.24(0.83,1.81)$ & .15 & $1.20(1.01,1.39)$ & .13 \\
\hline Fear of Scrutiny (Social Phobia Scale) & $0.99(0.77,1.40)$ & .04 & $1.13(0.77,1.60)$ & .09 & $1.11(0.76,1.63)$ & .08 \\
\hline
\end{tabular}

All variables were entered simultaneously, and all models controlled for gender, body mass index, age, and depression. Effect sizes reflect Cohen's d (small $=.20$, medium $=.50$, large $=.80$ )

$A N$ Anorexia Nervosa, BN Bulimia Nervosa, EDNOS Eating Disorders Not Otherwise Specified, SAD Social Anxiety Disorder

$* p<.001$

${ }^{\text {a }}$ Reference group

[for younger adolescent girls: $M=28.85, S D=15.95$; $t(491)=4.10, p<.001, d=.89$; for older adolescent girls: $M=36.47, S D=14.41 ; t(500)=5.78, p<.001$, $d=.94]$, yielding in both cases large effect sizes. Likewise, scores on the SAAS were significantly higher in younger $(M=39.22, S D=12.10)$ and older adolescent boys $(M=41.98, S D=11.20)$ with elevated EAT-26 scores than in those with lower scores [for younger adolescent boys: $M=27.57, S D=13.85 ; t(508)=2.65$, $p<.01, d=.80$; for older adolescent boys: $M=31.90$, $S D=12.92 ; t(488)=2.80, p<.01, d=.81]$, yielding large effect sizes in both cases.

\section{Study 2: ED Sample}

A CFA on the data for adolescents with EDs provided a good fit to the data (Model Ob in Table 3). Each SAAS item loaded significantly on the SAA latent factor; these standardized coefficients are reported in Table 1 . Thus, the results revealed that the one-factor structure of the SAAS was a good representation of the ED participants' responses as well.

The descriptive statistics, Cronbach's alpha $(\alpha)$ values, and the correlations among SAAS and the other measures for the sample of younger and older adolescent girls and boys with EDs are displayed in Table $2 .{ }^{13}$ In all sub-

\footnotetext{
13 There were no differences between ED diagnostic groups in SAAS score $[F(2700)=0.22, n s]$. Observed means for SAA by ED diagnostic groups were as follows: $55.48(S D=12.35)$ for $\mathrm{AN}$; $56.11(S D=11.88)$ for $\mathrm{BN}$, and; $55.33(S D=10.96)$ for EDNOS. The descriptive statistics of the SAAS for the entire ED sample and across diagnostic status (i.e., with or without comorbid SAD) are provided in the footnote (n. 14) of the present manuscript. For interested readers the descriptive statistics for the remaining study measures, and their associations with the SAAS stratified by ED diagnosis and diagnostic status are available from the corresponding author on request.
}

samples, $\alpha$ coefficients are above .90 , which indicates excellent internal consistency [64]. Moreover, the SAAS was moderately associated with depression severity, but strongly related to overall severity of ED psychopathology, fear of interacting in dyads and groups, fear of being scrutinized and negatively evaluated by others (Table 2 ). These results further support the convergent validity of the SAAS.

Finally, as seen in Table 4, that reports the results of logistic regressions with all domains of anxiety entered simultaneously (see footnote 9) only the SAAS differentiated each ED diagnostic group with comorbid SAD from each $\mathrm{ED}$ diagnostic group without $\mathrm{SAD}$, yielding strong effect sizes.

\section{Study 3: ME/I and Latent Mean Comparisons}

As noted in the data analytic plan, we used the combined data of Studies 1 and 2 to test the ME/I of the SAAS across groups (multi-group CFA) after assessing the model fit (i.e., single CFA) for each group separately. As shown in Table 3, which reports the fit indices, and the changes $(\Delta)$ in CFI, RMSEA, and SRMR, the 16-item model provided a good overall fit to the data for girls (Model 0c) and boys (Model 0d)-including clinical and non-clinical participants, younger (Model 0e) and older (Model 0f) adolescentsincluding clinical and non-clinical participants-, and ED participants with (Model $0 \mathrm{~g}$ ) and without comorbid SAD (Model 0h); for ease of presentation the results of the single CFAs (performed in Studies 1 and 2) on the data for the community sample of adolescents (Model 0a) and adolescents with EDs (Model Ob) are available in the same table. Also the evaluation of the configural factor loading, and intercept invariance models (Models 1-3 in Table 3) fit the data well, and when the equivalence of the factor loadings and intercept values were added, the changes in fit indices 
were within Chen's [68] recommendations for both metric (Model 2 vs. Model 1) and scalar invariance (Model 3 vs. Model 2) (see Table 3). Hence, the 16-item model of the SAAS was invariant across all groups. Given that scalar invariance was met, subsequently the latent mean differences across the above groups were evaluated; adolescents from community, boys, younger adolescents, and ED participants without SAD operated as the reference groups. Significant (all $p s<.001$ ) latent mean differences on the $\mathrm{SAA}^{14}$ latent factor were observed with the adolescents with EDs $(z=18.56, d=.93)$, girls $(z=16.31, d=.67)$, older adolescents $(z=5.75, d=.22)$, and ED participants with comorbid SAD $(\mathrm{z}=19.27, d=.97)$ endorsing higher scores.

\section{Discussion}

Consistent with prior research among English-speaking adults [21, 24], the results obtained in our Italian community and clinical sample of adolescents using single and multi-group CFAs upheld the unidimensional factor structure of the SAAS — not only for the entire samples but also as a function of gender, age categories, and diagnostic status (i.e., ED participants with and without comorbid SAD). Thus, the replicability as well as the consistency of the one-factor model in a non-English speaking setting and across different groups was supported. Our findings also extend prior research by (a) providing reliability evidence for the SAAS among adolescents as opposed to adults, and (b) more extensively verifying the construct (convergent and discriminant) validity [64] of the SAAS.

Psychometric analyses of the SAAS in both community and clinical samples of younger and older adolescent girls and boys showed excellent internal consistency reliability ( $\alpha$ s $=.93-.96)$ [64] for the Italian version of the SAAS. The Cronbach's alpha coefficients are comparable to those found for the original (English) version of SAAS ( $\alpha$ s $=.93-.95)$ [21, 24], suggesting that language differences did not compromise the effectiveness of the 16 SAAS items [44, 50]. The ICCs obtained (.81-.84) within a subset of 309 community adolescents indicated that the SAAS score was stable over a relatively short-term period (3-weeks) [64].

\footnotetext{
${ }^{14}$ Observed means for the SAA were: $39.87(S D=12.75)$ for boys (including clinical and non-clinical participants), $38.23(S D=15.88)$ for adolescents from community, $44.96(S D=10.62)$ for $\mathrm{ED}$ participants without SAD, $42.80(S D=14.48)$ for younger adolescents (including clinical and non-clinical participants), 48.65 $(S D=14.35$ ) for girls (including clinical and non-clinical participants), $50.30(S D=11.51)$ for adolescents with EDs, 55.64 $(S D=12.02)$ for ED participants with comorbid SAD, and 45.72 $(S D=11.96)$ for older adolescents (including clinical and nonclinical participants).
}

Evidence for the convergent validity for the Italian version of the SAAS was obtained across the first two studies by examining its relationship to body image, social avoidance, social distress, dieting, bulimia, oral control (i.e., subscales of the EAT-26 [55]), frequency of teasing about appearance (Study 1-community sample), other situational domains of social anxiety, severity of ED psychopathology (i.e., global score of the EDE-120D interview [48]), and depression (Study 2-ED sample). SAA has been found to occur concurrently with other relevant types of social fears among non-clinical adults [6, 9, 21, 24]. The strong correlations found among fear of scrutiny, fear of social interactions, fear of negative evaluation, and SAAS are in line with and extended previous findings to adolescents with EDs. Of additional note, the SAAS and BFNE scales were significantly inter-correlated ( $r s=.67-$ .69), but the strength of their association was not enough (i.e., $\geq .80$ ) to suggest substantial construct overlap [41, 64]. The correlation coefficients obtained in the ED sample are consistent with those obtained in prior research among non-clinical adults $[6,9,21]$, and all together suggest that SAA is distinct from fear of negative evaluation because it focuses specifically on fear of social judgments based on appearance versus more general fears of being negatively evaluated [21, 24]. Nevertheless, further investigation of this issue would be beneficial. The moderate association found between SAAS and severity of depression levels in this clinical adolescent sample is in line with previous findings using non-clinical samples [21, 24], even though the coefficients were typically higher in our ED sample. Although this is not surprising given the high levels of comorbidity of affective problems in ED patients with and without comorbid SAD [7], further research is needed to corroborate the suggestion that negative evaluation of one's appearance may affect mood states [21].

While research has long documented strong inter-relationships between social avoidance and distress and fear of negative evaluation, among community adolescents [34, 37, 69], our adolescent community data provided evidence that the SAAS is also strongly correlated with the above variables. These results may suggest that adolescents who are afraid of appearance-related evaluation not only experience social inhibition, distress, and discomfort in social situations or with unfamiliar people, but also behave in an avoidant way in social relationships in order to prevent potential negative evaluations [21, 24]. They deserve, however, further empirical attention within a clinical sample given that social avoidance, which is a key aspect of SAD related to behavioural inhibition [16], is high in all forms of EDs [70]. Furthermore, in the community sample the frequency of negative appearance-related feedback from significant others was strongly and significantly positively related to SAAS scores. This finding seems to 
support scholars' suggestion that teasing might produce or reinforce SAA [24, 39], but longitudinal studies using objective measures of teasing are needed to examine direction of causality, since the possibility that high SAA levels may lead to an increase in perceived teasing could not be ruled out [40].

In the community sample, the SAAS also appeared to be associated with body dissatisfaction assessed by the BIQ [54]. It should be noted, however, that SAAS correlation coefficients with the disparity between self-reported actual and ideal physical attributes, and associated emphasis on meeting physical ideals (BIQ composite score) were moderate, whereas the correlations were typically high with measures of social anxiety, distress and avoidance across all age and gender community subgroups (Table 2). The same picture emerged in the validation studies of the original SAAS that used different negative body image measures, social anxiety, and distress [24] (for details, see "introduction" section). Overall, prior and current results indicate that the SAAS falls within the larger class of social anxiety rather than negative body image.

It has been claimed that individuals who are or become anxious and concerned about being negatively evaluated by others because of their own appearance may be more driven to (a) control and restrict their quantity and/or quality of the food consumed in an attempt to anticipate and ward off potentially negative judgment and evaluation by others, and/or (b) engage in binge eating behaviours as a means of coping with aversive fears and feelings [6, 13, 22, 23, 36]. In Study 1, the strong association between SAAS scores and subscales of the EAT-26 [55] across all age and gender community groups are consistent with these hypotheses, and with the outcomes of both cross-sectional and longitudinal studies [6, 9, 11, 13]. Our data also indicated high correlations between SAAS scores and overall severity of ED psychopathology (assessed via a clinical interview) in the ED sample. Moreover, they revealed that: (a) SAAS discriminated community younger and older adolescent girls and boys with elevated and nonelevated (global) scores on the EAT-26 [55] (Study 1); (b) adolescent with EDs endorse higher scores on SAA latent factor than those from the community (Study 3), and; (c) the ED diagnostic groups did not differ in terms of SAAS levels. ${ }^{13}$ The latter finding is important given increasing efforts to identify underlying aetiological and maintenance processes of eating pathology regardless of specific diagnostic categorisation, and the efforts to develop common psychological assessment and interventions [4, 71-73]. Further research needs to concentrate on specific eating-disordered behaviours as well as diagnostic categories and subtypes among both adolescent and adult ED cases.

As a novel and incremental advance over previous work $[10,21,24]$, the results provide solid evidence that the SAAS is measurement invariant across all examined groups. That is, the same underlying factor structure exists in both clinical and community populations, independent of gender, age, and diagnostic status (i.e., EDs with and without comorbid SAD), and participants perceive, interpret, and respond to the content of the items in a similar way [41, 45]. As such, the SAAS may be used to compare levels of SAA across these samples with increased confidence. Indeed, according to Byrne [41], if one's interest is in the comparison of observed mean differences between groups, the demonstration of metric and scalar invariance are critical and sufficient. As noted, however, in the current work, once scalar invariance was tenable, the differences in the latent variable means were compared, since latent means are better indicators of true group differences as they are not associated with measurement error [41, 45]. Overall the latent mean comparisons showed that girls, older adolescents, adolescents with EDs, and ED participants with comorbid SAD report higher SAA than boys, younger adolescents, adolescents from community, and ED participants without SAD.

As in the current study, when the raw scores on the SAAS of the participants are compared, other studies have found [29, 30] that ED patients obtained a higher score than control community groups. Although, to our knowledge, no research has analysed the role of gender and age in the specific fear of judgment based on appearance (SAA) in adult or adolescent samples, the gender and age differences across adolescents found in this research converged with a large body of research indicating that in general terms body and overall appearance concerns, social fears and particularly negative evaluation fears, and social anxiety are more salient from mid-adolescence onwards and across female participants [26, 27, 32, 34, 37, 47, 62, 69]. Developmental explanations for higher levels of SAA occurring in older adolescents include strong emotional investment in peers and the increasing pressures of the peer group [32, 33, 35]. Future studies are necessary to substantiate these hypotheses, and further elucidate the role of gender and age in SAA, given the dearth of research on this topic and that in this work the effect sizes for age differences during adolescence were small $(d=.22)$.

The finding that ED participants with comorbid SAD scored higher on SAA than ED participants without SAD might be an important consideration for researchers and clinicians. SAD has the highest occurrence of all anxiety disorders in individuals with EDs [8, 17, 18, 20] and comorbid anxiety can interfere with the course and effectiveness of treatment [74, 75]. SAA may be essential to understand the high co-occurrence between EDs and SAD $[6,9,21,24]$ according to results of our multivariate logistic regressions (Table 4); the SAAS score had significantly higher odds ratios for the $\mathrm{AN}, \mathrm{BN}$ and EDNOS 
groups with SAD compared to AN, BN and EDNOS groups without comorbid SAD. Experimental manipulations of SAA revealed its unique effects on social anxiety, negative body image, and food intake in undergraduates [22]. Further, a recent study considering both SAA and more general fear of negative evaluation found that only SAA (as measured by the SAAS) was uniquely associated with both social anxiety and ED symptoms and acted as a shared risk factor for social anxiety and ED symptomatology in two independent samples of American collegeaged sample [9]. Although it would be ideal to assess and investigate SAA as potential vulnerability linking social anxiety with disordered eating in a clinical sample, prior findings along with those obtained here lend some credence to suggestion that SAA may represent a strong shared etiological vulnerability for both SAD and EDs [9, 22, 39]. ${ }^{15}$ If future studies fully substantiate the above hypothesis, then there exists the possibility for creating transdiagnostic interventions that ameliorate suffering from both disorders [9].

This collection of studies indicated that SAAS is a psychometrically sound instrument to assess SAA in both Italian community and ED samples of 11- to 18-year-old girls and boys. More research, however, is needed to examine the ability of the SAAS to discriminate community adolescents with high and low levels of social anxiety, and its predictive validity in terms of onset and maintenance of SAD and EDs. Future studies should also investigate the psychometric properties of the SAAS in non-Italian speaking adolescent samples and among individuals with SAD and other clinical samples (i.e., patients with body dysmorphic disorder and people with medical conditions affecting their appearance) and across ethnic groups. Furthermore, since most of our findings (especially from the non-clinical sample) are based on self-report measures, replication of our results by means of other measures, such as performance-based measures (e.g., attentional bias towards social cues) or information assessed by others (e.g., teachers, family members) may be useful. As the design of our studies precludes long term examination of the stability of the SAAS and of ME/I over time, future studies need to address these issues. How the SAAS affects additional

\footnotetext{
15 Our findings (Table 4) do not imply that the other domains of social anxiety considered (as measured by BFN, SPS, and SIAS) are not important for the co-occurrence of EDs with SAD. Rather, we believe that they are important given the results of univariate logistic regression models (footnote 10). However, the more specific form of fear of negative evaluation focusing on appearance (SAA) may be a unique construct of importance for the co-occurrence of EDs and SAD and what may drive the relationship between social anxiety and ED symptoms. This is consistent with what we found in the multivariate logistic regressions (Table 4), current vulnerability models for both EDs and SAD [9] (see also "introduction"), and other previous studies [22].
}

specific behaviours that are associated with concerns about appearance (i.e., compulsive exercising, or anabolic steroid use [76-82]) needs to be also elucidated. Finally, in this work the DSM-IV criteria for EDs and SAD [28] were used as data were collected before the official publication of the DSM-5 [83]. Therefore, future studies should adopt the new diagnostic criteria for evaluating the co-occurrence of the disorders.

\section{Summary}

The current collection of studies corroborates prior research on the psychometric properties of the original (English) SAAS among adults samples, and extends the research base by examining its reliability, factor, construct validity and ME/I in a large Italian community and $\mathrm{ED}$ sample of 11- to 18-year-old girls and boys. In addition, the current work constitutes the largest comprehensive investigation of the co-occurrence between SAD and EDs within an adolescent sample seeking treatment for an ED. It is also the first to ascertain to what extent SAA or other known social fears (fear of scrutiny, of negative evaluation, and of social interactions) related to ED psychopathology distinguish adolescents with an ED only from those with comorbid SAD. Overall, our findings indicated that SAAS is a measurement invariant instrument, which displays good psychometric properties and can be used in a rapid, easy manner as a tool for assessing SAA in both Italian community and ED samples of 11- to 18-year-old girls and boys. Significant between group differences on the SAA latent factor exist, demonstrating that adolescents with EDs, girls, older adolescents, and ED participants with comorbid SAD report higher SAA than adolescents from community, boys, younger adolescents, and ED participants without comorbid SAD. Further, this work provides evidence for the unique effects of SAA in differentiating ED patients (regardless the specific diagnosis) with and without comorbid SAD.

\section{References}

1. Neumark-Sztainer D, Wall M, Larson NI, Eisenberg ME, Loth K (2011) Dieting and disordered eating behaviors from adolescence to young adulthood: findings from a 10-year longitudinal study. J Am Diet Assoc 111(7):1004-1111

2. Dakanalis A, Timko CA, Carrà G, Clerici M, Zanetti MA, Riva G, Caccialanza R (2014) Testing the original and the extended dual-pathway model of lack of control over eating in adolescent girls. A two-year longitudinal study. Appetite 82:180-193

3. Riva G, Gaudio S, Dakanalis A (2015) The neuropsychology of self-objectification. Eur Psychol 20(1):34-43. doi:10.1027/10169040/a000190 
4. Dakanalis A, Carrà G, Calogero R, Zanetti MA, Gaudio S, Caccialanza R, Riva G, Clerici M (2014) Testing the cognitivebehavioural maintenance models across DSM-5 bulimic-type eating disorder diagnostic groups: a multi-centre study. Eur Arch Psychiatry Clin Neurosci. doi:10.1007/s00406-014-0560-2

5. Riva G, Gaudio S, Dakanalis A (2014) I'm in a virtual body: a locked allocentric memory may impair the experience of the body in both obesity and anorexia nervosa. Eat Weight Disord 19(1):133-134

6. Levinson CA, Rodebaugh TL (2012) Social anxiety and eating disorders. The role of negative social evaluation fears. Eat Behav $13: 27-35$

7. Hughes EK, Goldschmidt AB, Labuschagne Z, Loeb KL, Sawyer SM, Le Grange D (2013) Eating disorders with and without comorbid depression and anxiety: similarities and differences in a clinical sample of children and adolescents. Eur Eat Disord Rev 21:386-394

8. Pallister E, Waller G (2008) Anxiety in the eating disorders. Understanding the overlap. Clin Psychol Rev 28:366-386

9. Levinson CA, Rodebaugh TL, White EK, Menatti AR, Weeks JW, Iacovino JM, Warren CS (2013) Social appearance anxiety, perfectionism, and fear of negative evaluation. Distinct or shared risk factors for social anxiety and eating disorders? Appetite 67:125-133

10. Claes L, Hart TA, Smits D, Van Den Eynde F, Mueller A, Mitchell JE (2012) Validation of the social appearance anxiety scale in female eating disorder patients. Eur Eat Disord Rev 20:406-409

11. Dakanalis A, Carrà G, Calogero R, Fida R, Clerici M, Zanetti MA, Riva G (2014) The developmental effects of media-ideal internalization and self-objectification processes on adolescents' negative body-feelings, dietary restraint, and binge eating. Eur Child Adolesc Psychiatry. doi:10.1007/s00787-014-0649-1

12. Hinrichsen H, Waller G, van Gerko K (2004) Social anxiety and agoraphobia in the eating disorders: associations with eating attitudes and behaviours. Eat Behav 5:285-290

13. Dakanalis A, Clerici M, Caslini M, Favagrossa L, Prunas A, Volpato C, Riva G, Zanetti MA (2014) Internalization of sociocultural standards of beauty and disordered eating behaviours: the role of body surveillance, shame, and social anxiety. J Psychopathol 20(1):33-37

14. Dakanalis A, Zanetti AM, Riva G, Colmegna F, Volpato C, Madeddu F, Clerici M (2015) Male body dissatisfaction and eating disorder symptomatology: moderating variables among men. J Health Psychol 20(1):80-90. doi:10.1177/ 1359105313499198

15. Riva G, Gaggioli A, Dakanalis A (2013) From body dissatisfaction to obesity. How virtual reality may improve obesity prevention and treatment in adolescents. Stud Health Technol Inf 184:356-362

16. Hofman SG, Di Bartolo PM (2012) Social anxiety. Clinical, developmental, and social perspectives. Elsevier, New York

17. Godart NT, Flament MF, Curt F, Perdereau F, Lang F, Venisse JL, Halfon O, Bizouard P, Loas G, Corcos M, Jeammet P, Fermanian J (2003) Anxiety disorders in subjects seeking treatment for eating disorders: a DSM-IV controlled study. Psychiatry Res 117:245-258

18. Swinbourne J, Touz S (2007) The co-morbidity of eating disorders and anxiety disorders: a review. Eur Eat Disord Rev $15: 253-274$

19. Godart NT, Flament MF, Lecrubier Y, Jeammet P (2000) Anxiety disorders in anorexia nervosa and bulimia nervosa: comorbidity and chronology of appearance. Eur Psychiatry 15:38-45

20. Swinbourne J, Hunt C, Abbott M, Russell J, St Clare T, Touz S (2012) The comorbidity between eating disorders and anxiety disorders: prevalence in an eating disorder sample and anxiety disorder sample. Aust N Z J Psychiatry 46:118-131

21. Levinson CA, Rodebaugh TL (2011) Validation of the Social Appearance Anxiety Scale: factor, convergent, and divergent validity. Assessment 18:350-357

22. Levinson CA, Rodebaugh TL (2014) Negative social-evaluative fears produce social anxiety, food intake, and body dissatisfaction: evidence of similar mechanisms through different pathways. Clin Psychol Sci. doi:10.1177/2167702614548891

23. Rieger E, Van Buren D, Bishop M, Tanofsky-Kraff M, Welch R, Wilfley D (2010) An eating disorder-specific model of interpersonal psychotherapy (IPT-ED): causal pathways and treatment implications. Clin Psychol Rev 30:400-410

24. Hart TA, Flora DB, Palyo SA, Fresco DM, Holle C, Heimberg RG (2008) Development and examination of the Social Appearance Anxiety Scale. Assessment 15:48-59

25. Hart EA, Leary MR, Rejeski W (1989) The measurement of social physique anxiety. J Sport Exerc Psychol 11:94-104

26. Maïano C, Morin AJS, Monthuy-Blanc J, Garbarino J (2010) Construct validity of the fear of negative appearance evaluation scale in a community sample of French adolescents. Eur J Psychol Assess 26(1):19-27

27. Cash TF (2012) Encyclopedia of body image and human appearance. Elsevier, Oxford

28. American Psychiatric Association (2000) Diagnostic and statistical manual of mental disorders, 4 th edn, text revision. American Psychiatric Association, Washington

29. Koskina A, Van Den Eynde F, Meisel S, Campbell IC, Schmidt U (2011) Social appearance anxiety and bulimia nervosa. Eat Weight Disord 16:142-145

30. Van den Eynde F, Giampietro V, Simmons A, Uher R, Andrew CM, Harvey PO, Campbell IC, Schmidt U (2013) Brain responses to body image stimuli but not food are altered in women with bulimia nervosa. BMC Psychiatry 2013(13):302. doi:10.1186/ 1471-244X-13-302

31. Brunet J, Sabiston CM, Dorsch KD, McCreary DR (2010) Exploring a model linking social physique anxiety, drive for muscularity, drive for thinness and self-esteem among adolescent boys and girls. Body Image 7:137-142

32. Calogero RM, Thompson JK (2010) Gender and body image. In: Chrisler JC, McCreary DM (eds) Handbook of gender research in psychology, vol 2, 1st edn. Springer, New York, pp 153-184

33. Sebastian C, Burnett S, Blakemore S (2008) Development of the self-concept during adolescence. Trends Cogn Sc 12:441-446

34. Kearney CA (2005) Social anxiety and social phobia in youth. Characteristics, assessment, and psychological treatment. Springer, New York, NY

35. Parker JG, Rubin KH, Erath SA, Wojslawowicz JC, Buskirk AA (2006) Peer relationships, child development, and adjustment: a developmental psychopathology perspective. In: Cicchetti D, Cohen DJ (eds) Developmental psychopathology, vol 1, 2nd edn. Wiley, Hoboken, NJ, pp 419-493

36. Dakanalis A (2014) Body image measures and risk factors for EDs: a series of validation and longitudinal studies on community and clinical samples. PhD [dissertation], University of Pavia, Pavia, IT

37. Sumter SR, Bokhorst CL, Westenberg PM (2009) Social fears during adolescence: Is there an increase in distress and avoidance? J Anxiety Disord 23:897-903

38. Stein DJ, Ono Y, Tajima O, Muller JE (2004) The social anxiety disorder spectrum. J Clin Psychiatry 65:27-33

39. Bogels SM, Stein M (2009) Social Phobia: Towards DSM-V. In: Andrews G, Charney DS, Sirovatka PJ, Regier DR (eds) Stressinduced and fear circuitry disorders: advancing the research agenda for DSM-V, 1st edn. American Psychiatric Association, Arlington, pp 59-76 
40. Sweetingham R, Waller G (2008) Childhood experiences of being bullied and teased in the eating disorders. Eur Eat Disord Rev. 16(5):401-407

41. Byrne BM (2011) Structural equation modeling with Mplus: basic concepts, application and programming. Routledge, New York

42. Hagger MS, Aşçi FH, Lindwall M, Hein V, Mülazimoğlu-Balli O, Tarrant M, Ruiz YP, Sell V (2007) Cross-cultural validity and measurement invariance of the social physique anxiety scale in five European nations. Scand J Med Sci Sports 17:703-719

43. Oyserman D, Coon HM, Kemmelmeier M (2002) Rethinking individualism and collectivism: evaluation of theoretical assumptions and meta-analyses. Psychol Bull 128:3-72

44. Dakanalis A, Zanetti MA, Clerici M, Madeddu F, Riva G, Caccialanza R (2013) Italian version of the Dutch Eating Behavior Questionnaire. Psychometric proprieties and measurement invariance across sex. BMI-status and age. Appetite 71:187-195

45. Sass D (2011) Testing measurement invariance and comparing latent factor means within a confirmatory factor analysis framework. J Psychoeduc Assess 29(4):347-363

46. Dakanalis A, Riva G (2013) Mass media, body image and eating disturbances: The underlying mechanism through the lens of the objectification theory. In: Sams LB, Keels JA (eds) Handbook on body image: gender differences, sociocultural influences and health implications, 1st edn. Nova Science Publishers, New York, pp 217-235

47. Crocetti E, Hale WW, Fermani A, Raaijmakers Q, Meeus W (2009) Psychometric properties of the Screen for Child Anxiety Related Emotional Disorders (SCARED) in the general Italian adolescent population: a validation and a comparison between Italy and The Netherlands. J Anxiety Disord 23(6):824-829

48. Mannucci E, Ricca V, Di Bernardo M, Rotella CM (1996) Eating disorders and eating disorder examination. Il Diabete 8:127-131

49. Kaufman J, Birmaher B, Rao U, Ryan N (2004) Test K-SADS-PL Intervista diagnostica per la valutazione dei disturbi psicopatologici in bambini e adolescenti. Erickson, Trento

50. Van de Vijver F, Hambleton RK (1996) Translating tests. Some practical guidelines. Eur Psychol 1:89-99

51. Conti L (1999) Repertorio delle scale di valutazione in psichiatria - Tomo II. S.E.E, Firenze

52. Vallerand RJ (1989) Vers une méthodologie de validation transculturelle de questionnaires psychologiques: implications pour la recherche en langue française. Can Psychol 4:662-680

53. Matera C, Nerini A, Stefanile C (2013) The role of peer influence on girls' body dissatisfaction and dieting. Eur Rev Appl Psychol 63(2):67-74

54. Heron K, Smyth M (2013) Is intensive measurement of body image reactive? A two- study evaluation using ecological momentary assessment suggests not. Body Image 10(1):35-44

55. Dotti A, Lazzari R (1998) Validation and reliability of the Italian EAT-26. Eat Weight Disord 3:188-194

56. Saporetti G, Sancini S, Bassoli L, Castelli B, Pellai A (2004) Risk assessment for eating disorders in a high school: a study based on the Eating Attitudes Test 26. Minerva Pediatr 56(1):83-90

57. Sica C, Musoni I, Chiri LR, Bisi B, Lolli V, Sighinolfi C (2007) Social Phobia Scale and Social Interaction Anxiety Scale: their psychometric properties on Italian population. Appl Psychol Bull 252:59-71

58. Goldstein TR, Axelson DA, Birmaher B, Brent DA (2007) Dialectical behavior therapy for adolescents with bipolar disorder: a 1-year open trial. J Am Acad Child Adolesc Psychiatry 46:820-830

59. IBM Corp (2010) IBM SPSS statistics for windows, Version 19.0. IBM Corp, Armonk

60. Muthén LK, Muthén BO (1998-2011) Mplus User's Guide, 6th edn. Muthén \& Muthén, Los Angeles
61. Dolan CV (1994) Factor analysis of variables with 2, 3, 5 and 7 response categories: a comparison of categorical variable estimators using simulated data. Br J Math Stat Psychol 47:309-326

62. Bunnell BE, Joseph DL, Beidel DC (2013) Measurement invariance of the Social Phobia and Anxiety Inventory. J Anxiety Disord 27:84-91

63. Hu L, Bentler P (1999) Cutoff criteria for fit indices in covariance structure analysis: conventional criteria versus new alternatives. Struct Equ Model 6:1-55

64. Kaplan RM, Saccuzzo DP (2012) Psychological testing. Principles, applications \& issues, 8th edn. Wadsworth Cengage Learning, Belmont, CA

65. Cohen J (1992) A power primer. Psychol Bull 112:155-159

66. Allison PD (2012) Logistic regression using SAS: theory and application. SAS Institute Inc, Cary

67. SAS Institute Inc (2011) Base SAS 9.3 utilities: reference. SAS Institute Inc, Cary, NC

68. Chen FF (2007) Sensitivity of goodness of fit indices to lack of measurement invariance. Struct Equ Model 14:464-504

69. Ranta K, Junttila N, Laakkonen E, Uhmavaara A, La Greca AM, Niemi PM (2012) Social Anxiety Scale for Adolescents (SAS-A): measuring social anxiety among finnish adolescents. Child Psychiatry Hum Dev 43:574-591

70. Penas-Lledó E, Jiménez-Murcia S, Granero R, Penelo E, Agüera Z, Alvarez-Moya E, Fernández-Aranda F (2010) Specific eating disorder clusters based on social anxiety and novelty seeking. J Anxiety Disord 24:767-773

71. Dakanalis A, Timko CA, Zanetti MA, Rinaldi L, Prunas A, Carrà G, Riva G, Clerici M (2014) Attachment insecurities, maladaptive perfectionism, and eating disorder symptoms: a latent mediated and moderated structural equation modeling analysis across diagnostic groups. Psychiatry Res 215(1):176-184

72. Dakanalis A, Timko CA, Clerici M, Zanetti MA, Riva G (2014) Comprehensive examination of the trans-diagnostic cognitive behavioral model of eating disorders in males. Eat Behav 15(1):63-67

73. Tasca GA, Presniak MD, Demidenko N, Balfour L, Krysanski V, Trinneer A, Bissada H (2011) Testing a maintenance model for eating disorders in a sample seeking treatment at a tertiary care center: a structural equation modeling approach. Compr Psychiatry 52:678-687

74. Berkman ND, Lohr KN, Bulik CM (2007) Outcomes of eating disorders: a systematic review of the literature. Int J Eat Disord 40:292-309

75. Goodwin RD, Fitzgibbon ML (2002) Social anxiety as a barrier to treatment for eating disorders. Int J Eat Disord 32:103-106

76. Dakanalis A, Timko A, Madeddu F, Volpato C, Clerici M, Riva G, Zanetti AM (2015) Are the male body dissatisfaction and drive for muscularity scales reliable and valid instruments? J Health Psychol 20(1):48-59. doi:10.1177/1359105313498108

77. Dakanalis A, Zanetti MA, Riva G, Clerici M (2013) Psychosocial moderators of the relationship between body dissatisfaction and symptoms of eating disorders. A look a sample of young Italian women. Eur Rev Appl Psychol 63(5):323-334

78. Dakanalis A, Carrà G, Clerici M, Riva G (2015) Efforts to make clearer the relationship between body dissatisfaction and binge eating. Eat Weight Disord 20(1):145-146

79. Yean C, Benau EM, Dakanalis A, Hormes JM, Perone J, Timko CA (2013) The relationship of sex and sexual orientation to selfesteem, body shape satisfaction, and eating disorder symptomatology. Front Psychol. doi:10.3389/fpsyg.2013.00887

80. Dakanalis A, Riva G (2013) Current Considerations for Eating and Body-Related Disorders among Men. In: Sams LB, Keels JA (eds) Body image: gender differences, sociocultural influences and health implications, 1st edn. Nova Science Publishers, NewYork, pp 195-216 
81. Dakanalis A, Carrà G, Timko A, Volpato C, Pla-Sanjuanelo J, Zanetti A, Clerici M, Riva G (2015) Mechanisms of influence of body checking on binge eating. Int $\mathrm{J}$ Clin Health Psychol 15(2):93-104. doi:10.1016/j.ijchp.2015.03.003

82. Dakanalis A, Timko CA, Favagrossa L, Riva G, Zanetti MA, Clerici M (2014) Why do only a minority of men report severe levels of eating disorder symptomatology, when so many report substantial body dissatisfaction? Examination of exacerbating factors. Eat Disord 22(4):292-305. doi:10.1080/10640266.2014. 898980

83. American Psychiatric Association (2013) Diagnostic and statistical manual of mental disorders, 5th edn, (DSM-5). American Psychiatric Association, Washington 